\title{
Research on the Reliability Evaluation of Transmission Line on-Line Monitoring Device Based on the Comprehensive Evaluation Method
}

\author{
He Ye ${ }^{1,}$, , Liu Bing ${ }^{1}$, Chen Chao ${ }^{1}$, Zhang LiHong ${ }^{2}$, Pan $\mathrm{Bo}^{2}$, Xiong Wei ${ }^{2}$ \\ ${ }^{1}$ GuiZhou Power Grid Limited Liability Company Anshun Power Supply Bureau, Anshun, China \\ ${ }^{2}$ College of Electrical Engineering, GuiZhou University, Guiyang, China
}

Email address:

4785941@qq.com (He Ye),2605711477@qq.com (Zhang LiHong)

${ }^{*}$ Corresponding author

\section{To cite this article:}

He Ye, Liu Bing, Chen chao, Zhang LiHong, Pan Bo, Xiong Wei. Research on the Reliability Evaluation of Transmission Line on-Line Monitoring Device Based on the Comprehensive Evaluation Method. Science Discovery. Vol. 6, No. 3, 2018, pp. 199-204. doi: $10.11648 /$ j.sd.20180603.20

Received: April 19, 2018; Accepted: June 21, 2018; Published: June 26, 2018

\begin{abstract}
In view of the reliability of the on-line monitoring device for transmission lines, this paper puts forward a comprehensive evaluation method combining the analytic hierarchy process and the fuzzy evaluation method, in order to realize the reliability evaluation of the on-line monitoring device for transmission lines. Firstly, the structure of reliability evaluation system, which is composed of environmental factors, installation factors, human factors and management level factors, is established. Then, based on the evaluation system, the reliability of the on-line monitoring device for transmission lines is evaluated by fuzzy evaluation. Finally, according to the principle of maximum membership, the reliability of an on-line monitoring device for a transmission line is judged. The results show that the comprehensive evaluation method can well reflect the reliability of the on-line monitoring device for transmission lines, and provides a theoretical and practical basis for the reliability evaluation of the on-line monitoring device for transmission lines.
\end{abstract}

Keywords: Transmission Line, AHP, Fuzzy Evaluation Method, On-Line Monitoring Device, Reliability Assessment

\section{基于综合评价法的输电线路在线监测装置可靠性评估的研究}

何晔 ${ }^{*}$, 刘斌 ${ }^{1}$, 陈超 ${ }^{1}$, 张丽虹 $^{2}$, 潘波 $^{2}$, 熊炜 $^{2}$

1贵州电网有限责任公司安顺供电局, 安顺, 中国

${ }^{2}$ 电气工程学院, 贵州大学, 贵阳, 中国

邮箱

4785941@qq.com(何华),2605711477@qq.com(张丽虹)

摘要：针对输电线路在线监测装置可靠性工作的问题, 本文提出将层次分析法和模糊评价法相结合的综合评价法, 以 实现输电线路在线监测装置可靠性评估。首先建立了以环境因素、装置因素、人为因素和管理水平因素为组成的可靠 性评价体系结构; 然后在评价体系的基础上, 通过模糊评价对输电线路在线监测装置的可靠性进行评估; 最后根据最 大隶属度原则评判出某输电线路在线监测装置可靠性。结果证明, 综合评价法能够很好的反映输电线路在线监测装置 可靠性情况，为输电线路在线监测装置的可靠性评估提供了理论和实践依据。

关键词: 输电线路, 层次分析法, 模糊评价法, 在线监测装置, 可靠性评估 


\section{1. 引言}

随着智能电网技术的发展, 电力系统的结构和运行状 况愈发复杂, 电网供电的安全性和可靠性受到严重影响 [1-2]。输变电设备状态在线监测技术可以对电力系统的运 行状态进行实时监测, 一方面可以有效预防事故的发生, 另一方面当故障发生时，可以迅速做出处理，保障系统的 安全稳定运行。输电线路是电力系统的重要环节, 配置在 输电线路上的在线监测装置存在受环境影响大、相关技术 规范和要求不完善、监测数据不能共享等问题[3-6], 故对 其可靠性的评价显得尤为重要。

目前采用的可靠性评价方法主要有故障树分析法、事 件树分析法、层次分析法和模糊评价法等 [7-10]。其中, 故障树分析法和事件树分析法虽然简单明了、逻辑性强, 但构造故障树的多余量比较繁重, 复杂度高, 在进行逻辑 运算时很容易发生错误和漏判, 造成评价结果的可信度低。 层次分析法可以同时进行定性和定量分析[11-12], 简洁实 用, 所需的定量数据信息较少, 将各要素相对重要性的判 断转化为相对简单的权重进行计算。但由于定量数据少, 定性成分多, 得到的决策方案难以令人信服, 随着评价指 标和数据统计量的增多, 对于权重的确定是不小的考验, 并且特征值和特征向量的求法比较复杂, 计算结果的准确 性低[13]。模糊评价法是基于模糊数学理论, 能较好的解 决模糊的、难以量化的非确定性问题[14-15], 但其对指标 权重矢量的确定具有很强的主观性, 结果往往会出现超模 糊现象，造成评判的失败 [16]。综合以上方法的优缺点，
提出了将层次分析法和模糊评价法相结合的综合评价法, 该方法既能针对多个因素变量进行定性和定量分析, 又能 通过层次化的分析过程将每个因素对评判结果的影响程 度进行量化。

本文基于综合评价法对输电线路在线监测装置的可 靠性进行评估, 建立了以环境因素、装置因素、人为因素 和管理水平因素为可靠性指标的评价体系, 通过分析和评 价, 证明了该方法是一种应用于输电线路在线监测装置可 靠性评估的有效手段。

\section{2. 可靠性评价体系的构建}

输电线路在线监测装置可靠性评价体系可分为三层: 评价目标层、评价准则层和指标系数层。其中, 评价目标 层为输电线路在线监测装置的可靠性评价结果; 评价准则 层为四个维度指标, 即环境因素、装置因素、人为因素和 管理水平因素, 这四种因素对输电线路在线监测装置的可 靠性影响最大; 指标系数层为各二级指标, 各因素指标是 根据输电线路长期运行的状态数据总结归纳得出。其中环 境因素指标包括温度因素、湿度因素、电磁效应因素和辐 射干扰因素; 装置因素指标包括电源因素、主/子站因素、 通信因素和传感器因素; 人为因素指标包括技能因素、素 质因素、教育培训因素和身体因素; 管理水平因素包括监 管因素、领导因素、规章因素和生产责任因素。可靠性评 价体系结构见图1。

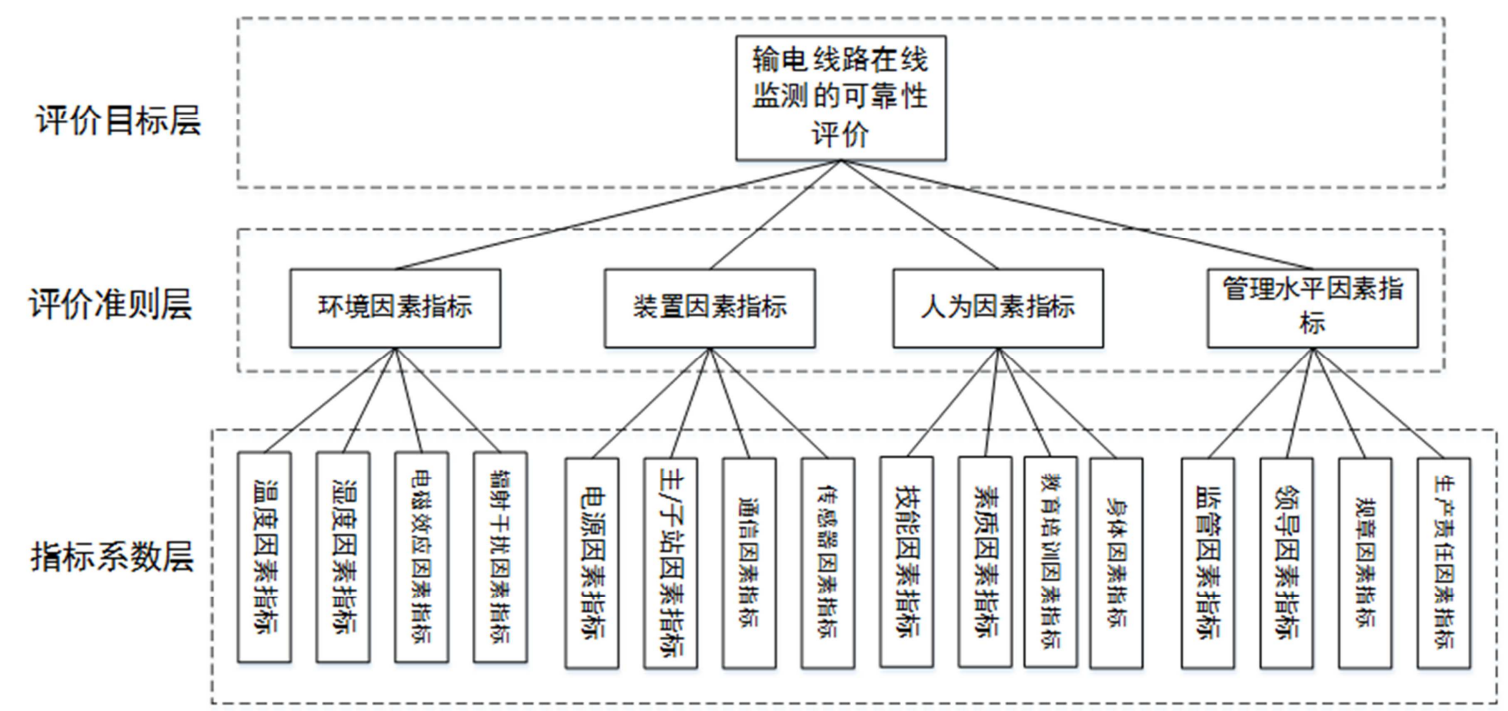

图1 输电线路可靠性评价体系结构。

\section{1. 层次的构建}

通过对输电线路在线监测装置可靠性各影响因素的 分析, 将这些因素划分为层次结构模型, 故该可靠性评价 体系结构分为环境因素、装置因素、人为因素和管理水平 因素四部分, 根据各因素的属性和关系可决定其所在的层 次，上一层次的因素对下一层次的因素起支配作用 [17]。

\section{2. 比较判断矩阵的构建及最大特征值求解}

应用层次分析法时, 首先要把问题条理化、层次化, 构造出一个层次分析结构模型。建立层次分析模型后, 就 可以在各层元素中进行两两比较构造出比较判断矩阵。假 定上一层的元素作为准则, 其对下一层的元素有支配关系, 即针对该准则, 将同一层两元素的重要性进行比较并赋予 
一定的数值, 构造出比较判断矩阵, 本文选取某地区输电 线路进行研究。

对于 $\mathrm{n}$ 个元素来说, 我们得到两两比较判断矩阵: $C=\left(c_{i j}\right)_{n \times n}$, 其中 $c_{i j}$ 表示元素 $\mathrm{i}$ 和因数 $\mathrm{j}$ 相对于目标的重要 值。

在层次分析法中, 为了使决策定量化, 形成比较判断 矩阵常根据1-9标度法[18-19]将判断定量化。

表1 判断矩阵标度及其含义。

\begin{tabular}{lll}
\hline 序号 & 重要性等级 & $c_{i j}$ 赋值 \\
\hline 1 & i, j两元素同等重要 & 1 \\
2 & i元素比 j元素稍微重要 & 3 \\
3 & i元素比 $\mathrm{j}$ 元素明显重要 & 5 \\
4 & i元素比 j元素强烈重要 & 7 \\
5 & i元素比 $\mathrm{j}$ 元素极端重要 & 9 \\
6 & i元素比 j元素稍微不重要 & $1 / 3$ \\
7 & i元素比元素明显不重要 & $1 / 5$ \\
8 & i元素比 j元素强烈不重要 & $1 / 7$ \\
9 & i元素比 $\mathrm{j}$ 元素极端不重要 & $1 / 9$ \\
\hline
\end{tabular}

评价目标层的两两判断矩阵可根据“专家经验”确定 出各层因素对上层的影响程度而建立, 如式1所示。

$$
C=\left[\begin{array}{cccc}
1 & 3 & 9 & 5 \\
1 / 3 & 1 & 3 & 7 \\
1 / 9 & 1 / 3 & 1 & 1 \\
1 / 5 & 1 / 7 & 1 & 1
\end{array}\right]
$$

构造出上述的比较判断矩阵后, 即可对判断矩阵进行 单排序计算。层次单排序计算问题可归结为计算判断矩阵 的最大特征值及其特征向量的问题 [20-21], 一般采用和积 法进行计算。其具体步骤如下:

1) 将判断矩阵的每一列元素作归一化处理:

$$
\bar{C}_{i j}=\frac{C_{i j}}{\sum_{1}^{n} C_{i j}}(i=1,2, \ldots, n)
$$

2) 将每一列经归一化处理后的判断矩阵按行相加, 即 为:

$$
\bar{W}_{i}=\sum_{1}^{n} \bar{C}_{i j}(i=1,2, \ldots, n)
$$

3) 对向量进行归一化处理:

$$
W_{i}=\frac{\bar{W}_{i}}{\sum_{1}^{n} \bar{W}_{j}}(i=1,2, \ldots, n)
$$

所得的向量 $W=\left(W_{1}, W_{2}, \ldots, W_{n}\right)$ 即为所求的特征向量 的近似解。评价目标层判断矩阵对应最大特征值的特征向 量如式5所示。

$$
\boldsymbol{W}=\left[\begin{array}{l}
0.5758 \\
0.2819 \\
0.0715 \\
0.0709
\end{array}\right]
$$

4) 计算判断矩阵的最大特征值

$$
\lambda_{\max }=\sum_{i=1}^{n} \frac{(A W)_{i}}{n W_{i}}
$$

其中 $(A W)_{i}$ 表示向量 $A W$ 的第 $\mathrm{i}$ 个元素。

求得评价目标层判断矩阵的最大特征值为: $\lambda_{\text {max }}=4.2014$ 。

\section{3. 一致性检验}

当判断矩阵不能保证具有完全一致时, 相应判断矩阵 的特征根也将发生变化, 这样就可以用判断矩阵特征根的 变化来检验判断的一致性程度[22]。

一致性指标 $C I$ 可以表示为:

$$
C I=\frac{\lambda_{\max }-n}{n-1}
$$

其中 $\lambda_{\text {max }}$ 为最大特征值, $\mathrm{n}$ 为指标元素的个数。

随机一致性指标 $C R$ 为:

$$
C R=\frac{C I}{R I}
$$

其中 $R I$ 为平均一致性指标, 可查表得到。

当 $C R<0.1$ 时, 说明所提判断矩阵满足一致性要求, 同 时说明指标的重要性排序是合理的。

可求得本线路评价目标层判断矩阵的 $C I=0.0671$, $C R=0.0754$, 此矩阵的一致性是可以接受的。

\section{3. 指标权重的确定}

\section{1. 评价目标层指标权重的确定}

当判断矩阵的一致性校验通过之后, 相应指标的权重 等于矩阵的最大特征根对应的特征向量, 矩阵阶数较大时, 常采用计算机辅助计算。若得出的权重结构不满意, 可以 直接设定专家认为有意义的权重值[23]。

评价目标层的指标权重根据 1.2 求得的最大特征值对 应的特征向量来确定, 由于其判断矩阵符合一致性要求, 故进一步认为计算所得矩阵特征向量 $W$ 即为对应的四个 维度指标的权重, 然后在实际的情况中根据收资数据普算 再进行权重微调, 各指标如表2 所示 (保留两位有效数字)。

表2 评价目标层四维度指标权重。

\begin{tabular}{lllll}
\hline $\begin{array}{l}\text { 对应维度 } \\
\text { 指标 }\end{array}$ & $\begin{array}{l}\text { 环境因素 } \\
\text { 指标 }\end{array}$ & $\begin{array}{l}\text { 装置因素 } \\
\text { 指标 }\end{array}$ & $\begin{array}{l}\text { 人为因素 } \\
\text { 指标 }\end{array}$ & $\begin{array}{l}\text { 管理水平 } \\
\text { 因素指标 }\end{array}$ \\
\hline $\begin{array}{l}\text { 原 始 对 应 } \\
\text { 权重 }\end{array}$ & 0.58 & 0.28 & 0.07 & 0.07 \\
$\begin{array}{l}\text { 微 调 后 对 } \\
\text { 应权重 }\end{array}$ & 0.57 & 0.28 & 0.07 & 0.07 \\
\hline
\end{tabular}




\section{2. 评价准则层指标权重的确定}

为求得准则层指标的权重, 首先需要求得各影响因素 最大特征值对应的特征向量, 即四个维度指标下对应的二 级指标构成的两两判断矩阵的特征向量, 并校验其一致性, 然后确定各影响因素的权重值。各影响因素的特征向量如 表3所示, 各影响因素的权重如表4所示。

表3 评价准则层各影响因素的特征向量。

\begin{tabular}{lllll}
\hline 维度指标 & 特征向量 & & & \\
\hline 环境因素 & 0.2310 & 0.1756 & 0.4300 & 0.1634 \\
装置因素 & 0.2387 & 0.1856 & 0.4276 & 0.1481 \\
人为因素 & 0.2471 & 0.1585 & 0.4359 & 0.1585 \\
管理水平因素 & 0.2074 & 0.1767 & 0.3756 & 0.2403 \\
\hline
\end{tabular}

准则层求得的特征向量所对应的最大特征值 $\lambda_{\text {max }}$ 分 别为 $4.0812,4.2354,4.1545,4.2425$ 。对其进行一致性校 验, 可分别得到 $C R$ 为 $0.0301,0.0882,0.0579,0.0908$, 均符合一致性要求, 故可由特征向量来确定权重, 准则层 各因素指标的权重如表4所示。

表4 评价准则层各因素对应的权重。

\begin{tabular}{lllll}
\hline 维度指标 & 对应权重 & & & \\
\hline 环境因素 & 0.228 & 0.173 & 0.426 & 0.173 \\
装置因素 & 0.225 & 0.179 & 0.438 & 0.158 \\
人为因素 & 0.228 & 0.153 & 0.452 & 0.167 \\
管理水平因素 & 0.207 & 0.176 & 0.375 & 0.242 \\
\hline
\end{tabular}

\section{4. 可靠性评价}

输电线路在线监测装置的可靠性评价一般采用模糊 评价的方法, 即利用模糊数学理论, 对受到多个因素指标 影响的输电线路, 按照一定的评判标准, 给出其获得某个 评语的可能性[24]。
首先确定指标系数层对评价准则层的单因素影响评 价矩阵 (如式9所示), 即模糊隶属度值矩阵。然后, 将 该矩阵与上一章求得的权重值做乘法, 此时得到的矩阵为 评价准则层的模糊评价矩阵, 如式10所示。

$$
\begin{gathered}
\boldsymbol{M}_{i}=\left[\begin{array}{cccc}
\boldsymbol{m}_{i 11} & \boldsymbol{m}_{i 12} & \ldots & \boldsymbol{m}_{i 1 n} \\
\boldsymbol{m}_{i 21} & \boldsymbol{m}_{i 22} & \ldots & \boldsymbol{m}_{i 2 n} \\
\ldots & \ldots & \ldots & \ldots \\
\boldsymbol{m}_{i j 1} & \boldsymbol{m}_{i j 2} & \ldots & \boldsymbol{m}_{i j n}
\end{array}\right] \\
M=\left[\begin{array}{c}
\boldsymbol{B}_{1} \boldsymbol{M}_{1} \\
\boldsymbol{B}_{2} \boldsymbol{M}_{2} \\
\boldsymbol{B}_{3} \boldsymbol{M}_{3} \\
\boldsymbol{B}_{4} \boldsymbol{M}_{4}
\end{array}\right]
\end{gathered}
$$

式中, $B_{i}(i=1,2,3,4)$ 为各因素指标权重。

最后求得评价目标层的综合评判:

$$
A=B M
$$

\section{1. 单因素影响评价矩阵的确定}

单因素影响评价矩阵即为各因素模糊隶属度值的确 定, 模糊隶属度函数的确定一般有模糊统计法、例证法、 专家经验法和二元对比排序法四种[25]。在对输电线路在 线监测装置可靠性的评价中, 由专家根据经验, 参考运行 监测的历史数据, 给出相应的评分, 得到评判矩阵。

表 5 为某条输电线路在线监测装置因素指标的隶属度 值, 该值是采用模糊统计法和专家经验法相结合的方法得 出, 据此可得出装置因素指标的评价矩阵, 如式12所示。

$$
M_{2}=\left[\begin{array}{cccc}
0.38 & 0.38 & 0.17 & 0.20 \\
0.21 & 0.30 & 0.15 & 0.23 \\
0.66 & 0.34 & 0.71 & 0.30 \\
0.52 & 0.28 & 0.34 & 0.47
\end{array}\right]
$$

\begin{tabular}{|c|c|c|c|c|c|c|}
\hline \multirow{2}{*}{ 评价准则层 } & \multirow{2}{*}{ 指标系数层 } & \multirow{2}{*}{ 评价目标层 } & \multicolumn{4}{|c|}{ 评语等级 } \\
\hline & & & 1 & 2 & 3 & 4 \\
\hline \multirow{16}{*}{ 装置因素指标 } & \multirow{4}{*}{ 电源因素 } & 供电电源的使用寿命是否较长 & $\sqrt{ }$ & $\sqrt{ }$ & $\sqrt{ }$ & \\
\hline & & 运行状态下电源因素的影响 & & & & $\sqrt{ }$ \\
\hline & & 未运行状态下电源因素的影响 & $\sqrt{ }$ & $\sqrt{ }$ & & \\
\hline & & 隶属度计算 & 0.38 & 0.38 & 0.17 & 0.20 \\
\hline & \multirow{4}{*}{ 主/子站因素 } & 主/子站软硬件设施是否完好 & & & $\sqrt{ }$ & \\
\hline & & 运行状态下主/子站因素的影响 & $\sqrt{ }$ & & & \\
\hline & & 未运行状态下主/子站因素的影响 & & $\sqrt{ }$ & & $\sqrt{ }$ \\
\hline & & 隶属度计算 & 0.21 & 0.30 & 0.15 & 0.23 \\
\hline & \multirow{4}{*}{ 通信因素 } & 通信设施及线路是否完好 & $\sqrt{ }$ & & $\sqrt{ }$ & \\
\hline & & 运行状态下通信因素的影响 & & $\sqrt{ }$ & $\sqrt{ }$ & $\sqrt{ }$ \\
\hline & & 未运行状态下通信因素的影响 & $\sqrt{ }$ & & $\sqrt{ }$ & $\sqrt{ }$ \\
\hline & & 隶属度计算 & 0.66 & 0.34 & 0.71 & 0.30 \\
\hline & \multirow{4}{*}{ 传感器因素 } & 传感器装置是否完好 & & & $\sqrt{ }$ & $\sqrt{ }$ \\
\hline & & 运行状态下传感器因素的影响 & $\sqrt{ }$ & $\sqrt{ }$ & & \\
\hline & & 未运行状态下传感器因素的影响 & $\sqrt{ }$ & & $\sqrt{ }$ & $\sqrt{ }$ \\
\hline & & 隶属度计算 & 0.52 & 0.28 & 0.34 & 0.47 \\
\hline
\end{tabular}

表5 装置因素隶属度计算。 
从而, 根据公式可得模糊评价矩阵:

$$
A_{1}=B_{1} M_{1}=(0.3871,0.2569,0.2281,0.379)
$$

同理, 可分别求得环境因素、人为因素和管理水平因 素的模糊评价矩阵, 如式14-16所示。

环境因素的模糊评价矩阵:

$$
A_{2}=B_{2} M_{2}=(0.4941,0.3318,0.4260,0.295)
$$

人为因素的模糊评价矩阵:

$$
A_{3}=B_{3} M_{3}=(0.2562,0.3338,0.4026,0.2931)
$$

管理水平因素的模糊评价矩阵:

$$
A_{4}=B_{4} M_{4}=(0.4901,0.1992,0.2306,0.320)
$$

\section{2. 可靠性模糊评价}

根据评价模型, 将评价指标层的权重与评价指标层的 隶属度值相乘和得到评价准则层的模糊隶属度值, 如式17 所示。

$$
\boldsymbol{M}=\left(\boldsymbol{A}_{1}, \boldsymbol{A}_{2}, \boldsymbol{A}_{3}, \boldsymbol{A}_{4}\right)=\left[\begin{array}{cccc}
0.3871 & 0.2569 & 0.2281 & 0.397 \\
0.4941 & 0.3318 & 0.426 & 0.295 \\
0.2562 & 0.3338 & 0.4026 & 0.2931 \\
0.4901 & 0.1992 & 0.2306 & 0.320
\end{array}\right]
$$

则评价目标层的隶属度值如下式所示, 根据此值对可 靠性进行综合评判。

$$
A=B M=(0.4155,0.2794,0.2967,0.3552)
$$

隶属度最大的值为 0.4155 , 根据模糊理论最大隶属度 原则, 该数值所对应的状态即为输电线路在线监测装置可 靠性评价体系所处的状态, 由此判定该输电线路在线监测 装置的可靠性属于中等水平, 再根据各因素指标所占的权 重, 说明在线监测装置的工作性能和管理水平有待进一步 提高, 同时, 应提高输电线路应对恶劣环境的能力, 并加 强对操作管理人员的教育培训。

\section{5. 结论}

本文针对输电线路在线监测装置的可靠性问题, 构建 了可靠性评价体系，并利用综合评价法对可靠性进行评估。 首先采用层次分析法和模糊评价法相结合的综合评价法 构建了以评价目标层、评价准则层和指标系数层为组成的 可靠性评价体系结构; 通过分析影响输电线路在线监测装 置可靠性的因素指标, 并运用层次分析法分别对评价目标 层和评价准则层的权重进行计算, 为可靠性的评价提供依 据; 最后采用模糊评价方法, 根据隶属度最大的原则, 对 输电线路在线监测装置可靠性状态进行评估, 并据此提出 改进措施, 为输电线路在线监测装置的可靠性评估提供了 理论和实践依据。

\section{致谢}

本文为南方电网科技项目(GZKJXM20170140)的阶段 性成果之一。

\section{参考文献}

[1] 徐望圣,童干,汪旭旭,秦冰.输电线路在线监测装置设备可靠 性的提升措施研究[J].电气开关,2016,54(03):19-21+26。

[2] 高晓婧.基于模糊综合评判的输电线路在线监测装置综合 评估[D].华北电力大学(北京),2016。

[3] 段满银,张国华.国内外直流输电系统可靠性指标对比分析 [J].电网技术, $2013,09: 2390-2395$ 。

[4] 曾庆禹.特高压交直流输电系统可靠性分析[J].电网技 术, 2013,10:2681-2688。

[5] 马为民,杨志栋,李亚男. $\pm 800 \mathrm{kV}$ 特高压直流输电工程共用接 地极的可靠性评价[J].高电压技术,2010,02:301-305。

[6] 张禄琦,周家启,刘洋, 谢开贵, 刘威,金小明.高压直流输电工 程可靠性指标统计分析[J].电力系统自动化,2007,19:95-99。

[7] 明娇,刘代勇.基于改进层次模糊评判的地区电网 AVC系统 告警信息分级模型 $[\mathrm{J}]$. 电力系统保护与控制, 2011,39(2):51-54。

[8] 王钦, 文福拴, 刘敏, 等.基于模糊集理论和层次分析法的 电力市场综合评价 $[\mathrm{J}]$. 电力系统自动化, 2009, 33（7）: 32-37。

[9] 满若岩, 付忠广. 基于模糊综合评判的火电厂状态评估 [J]. 中国电机工程学报, 2009, 29（5）：5-10。

[10] 何迎春.基于故障树的架空输电线路可靠性评估方法研究 [D].重庆大学,2012。

[11] 段若晨,王丰华,顾承昱,满玉岩,傅正财,刘亚东. 采用改进层 次分析法综合评估 $500 \mathrm{kV}$ 输电线路防雷改造效果 $[\mathrm{J}]$. 高电压 技术,2014,01:131-137。

[12] 黄知超,谢霞,王斌. 结合模糊综合评判与决策的电力系统状 态估计[J].电力系统保护与控制,2015,43(07):65-69。

[13] 郄晓和.基于灰色模糊理论的电力工程项目风险评价研究 [D].华北电力大学 (北京),2011。

[14] 李沝永. 基于模糊一神经网络的电力企业核心竞争力评价 研究[D].河北工程大学,2011。

[15] 湛顶,董元成.多因子分层模糊评价法在输电线路防雷改造 中的研究 [J].电气开关,2018,56(01):9-12+17。

[16] 张科伟,施泉生. 电力企业危机管理能力的模糊评价 [J]. 华 东电力, 2008,08:141-143。

[17] 周二雄, 李凤婷, 朱贺.基于改进层次分析法 (AHP) 的微 网-成本-效益评估 $[\mathrm{J}]$. 电力建设, 2013，34（3）：1-6。 
[18] 黎祚,周步祥,李君,林楠,李阳,刘金华. 考虑权重标度法的中 长期负荷预测综合模型 [J]. 电力系统及其自动化学 报,2013,05:96-99。

[19] 黄敬尧,杨爽,郭吴,李维维. 基于层次分析法的带电跨越方 式选择 $[J]$.电力建设,2013,05:106-109。

[20] 彭汝华.基于层次分析法的电力需求侧管理研究[D].重庆大 学,2008。

[21] 张晶晶,许修乐,丁明,李金忠,王健一,吴超.基于模糊层次分 析法的变压器状态评估 [J]. 电力系统保护与控 制,2017,45(03):75-81。
[22] 王丽丽. 模糊数学法结合层次分析法用于清洁生产潜力评 估研究[D].重庆大学,2010。

[23] 梁丁相, 陈曦.基于模糊综合评判理论的电力信息系统安全 风险评估模型及应用[J].电力系统保护与控制,2009,37 (5): 61-64。

[24] 刘瑾,冯瑛敏,章辉,冯亮,孙瑞雪,王丽珠.基于熵值分析和层 次分析法的智能电网电力终端接入网综合效益评价 [J]. 电 力建设,2015,05:136-140。

[25] 石雪. 电力市场运营规则评价指标体系及综合评价方法研 究[D]. 华北电力大学 (北京),2009。 